\title{
ON THE EXTENSION PROPERTY OF DILATATION MONOTONE RISK MEASURES
}

\author{
MASSOOMEH RAHSEPAR AND FOIVOS XANTHOS
}

\begin{abstract}
Let $\mathcal{X}$ be a subset of $L^{1}$ that contains the space of simple random variables $\mathcal{L}$ and $\rho: \mathcal{X} \rightarrow(-\infty, \infty]$ a dilatation monotone functional with the Fatou property. In this note, we show that $\rho$ extends uniquely to a $\sigma\left(L^{1}, \mathcal{L}\right)$ lower semicontinuous and dilatation monotone functional $\bar{\rho}: L^{1} \rightarrow(-\infty, \infty]$. Moreover, $\bar{\rho}$ preserves monotonicity, (quasi)convexity, and cashadditivity of $\rho$. Our findings complement recent extension results for quasiconvex law-invariant functionals proved in [17, 20]. As an application of our results, we show that transformed norm risk measures on Orlicz hearts admit a natural extension to $L^{1}$ that retains the robust representations obtained in 4, 6].
\end{abstract}

\section{Introduction And Notations}

In the axiomatic theory of risk measures, it is customary to assume that risk measures are defined on a subspace $\mathcal{X}$ of $L^{1}$. The problem of extending the domain of risk measures to the entire $L^{1}$ arises naturally in applications and has received significant attention in the mathematical finance literature (see e.g. [2, 10, 12, 15, 17, 20]). The celebrated work [10], asserts that if $\mathcal{X}$ is some $L^{p}$-space for $1 \leq p \leq \infty$, then any law-invariant convex risk measure $\rho: L^{p} \rightarrow(-\infty, \infty]$ with the Fatou property can be extended uniquely to a law-invariant and $\|\cdot\|_{1}$ lower semicontinuous convex risk measure $\bar{\rho}: L^{1} \rightarrow(-\infty, \infty]$. This result has been recently generalized to cover the case where $\mathcal{X}$ is an Orlicz space or a more general rearrangement invariant space ([2, 12, 17, 20]). A crucial step in the proof of the aforementioned results is that the underlying assumptions force the risk measure to be dilatation monotone. This property was introduced in [16] and reflects the general belief that balancing out should never increase the involved risk. For a connection with other notions of risk aversion, we refer the reader to [5, 17, 18, 19].

In this paper, we study the extension problem for dilatation monotone risk measures defined on a subset $\mathcal{X}$ of $L^{1}$. The sole assumption we impose on the domain is that $\mathcal{X}$ contains the space of simple random variables $\mathcal{L}$. This broad framework is motivated by the general class of transformed norm risk measures $([3,4])$, as in this case the domain typically is not a vector space (see Example 10). In Section 2, we gather the main results of the paper. Lemma 2 contains a result on convergence of finite conditional expectations that is of independent interest. In Proposition 3, we show that the dilatation monotonicity property coupled with the Fatou

Date: February 28, 2020.

2010 Mathematics Subject Classification. 91B30, 91G80, 46E30.

Key words and phrases. Extension of risk measures, dilatation monotonicity, law invariance, Fatou property, Orlicz spaces, transformed norm risk measures, higher order dual risk measures, dual representations, Kusuoka representations.

This research was supported by an NSERC Discovery Grant. This first author is also supported by an Ontario Graduate Scholarship. 
property implies that the underlying risk measure is lower semicontinuous with respect to the relative $\sigma\left(L^{1}, \mathcal{L}\right)$ topology. Theorem 4 is our main extension result. In Corollary 6 , we enlarge the class of domains $\mathcal{X}$ for which the extension problem for quasiconvex law-invariant risk measures has a positive solution. Section 3 contains our application to transformed norm risk measures $T$. In Theorem 12, we calculate the extension $\bar{T}: L^{1} \rightarrow(-\infty, \infty]$ and show that $\bar{T}$ preserves the desirable properties of $T$. In Corollary 13, we extend the dual representation of $T$ obtained in [4] and in Corollary 14, we extend the Kusuoka representation for higher order dual risk measures obtained in [6].

Notations. Let $(\Omega, \mathcal{F}, \mathbb{P})$ be a fixed nonatomic probability space. We denote by $L^{1}$ the space of integrable random variables on $(\Omega, \mathcal{F}, \mathbb{P})$ modulo almost sure equality and by $\|\cdot\|_{1}$ the $L^{1}$-norm. Throughout this paper, all equalities and inequalities are understood in the $\mathbb{P}$-almost sure (a.s.) sense. The space of bounded random variables is denoted by $L^{\infty}$. A subspace $\mathcal{X}$ of $L^{1}$ is said to be an ideal of $L^{1}$ whenever $|X| \leq|Y|$ and $Y \in \mathcal{X}$ imply $X \in \mathcal{X}$ for all $X \in L^{1}$. For any subset $\mathcal{S}$ of $L^{1}$, we denote by $\mathcal{I}(\mathcal{S})$ the smallest ideal of $L^{1}$ including $\mathcal{S}$, which is given by $\mathcal{I}(\mathcal{S})=$ $\left\{X \in L^{1}: n \in \mathbb{N}\right.$, there exist $X_{1}, \ldots, X_{n} \in \mathcal{S}$ and $\lambda_{i} \in \mathbb{R}_{+}$such that $\left.|X| \leq \sum_{i=1}^{n} \lambda_{i}\left|X_{i}\right|\right\}$ (see e.g. [1], p. 322). For any $A \in \mathcal{F}$, we denote the indicator function by $\mathbb{1}_{A}$. A random variable is simple if it has the form $\sum_{i=1}^{n} a_{i} \mathbb{1}_{A_{i}}$, where $n \in \mathbb{N}, a_{i} \in \mathbb{R}$ and $A_{i} \in \mathcal{F}$. The space of simple random variables is denoted by $\mathcal{L}$. We write $\pi$ to denote a finite measurable partition of $\Omega$ whose members have non-zero probabilities, and write $\Pi$ for the collection of all such $\pi$. We denote by $\sigma(\pi)$, the finite $\sigma$-subalgebra generated by $\pi$, and write $\mathbb{E}[X \mid \pi]:=\mathbb{E}[X \mid \sigma(\pi)]$ to denote the conditional expectation of $X \in L^{1}$ with respect to $\sigma(\pi)$. We recall below a few fundamental facts about conditional expectations. For any $\pi=\left\{A_{1}, \ldots, A_{k}\right\} \in \Pi$ and $X \in L^{1}$, we have that $\mathbb{E}[X \mid \pi]=\sum_{i=1}^{k} \frac{\mathbb{E}\left[X \mathbb{1}_{A_{i}}\right]}{\mathbb{P}\left(A_{i}\right)} \mathbb{1}_{A_{i}}$ and $\|\mathbb{E}[X \mid \pi]\|_{1} \leq\|X\|_{1}$. Moreover, $\mathbb{E}\left[X_{n} \mid \pi\right] \stackrel{\|\cdot\|_{1}}{\longrightarrow} \mathbb{E}[X \mid \pi]$ for every sequence $\left(X_{n}\right)_{n \in \mathbb{N}} \subset L^{1}$ such that $X_{n} \stackrel{\|\cdot\|_{1}}{\longrightarrow} X$.

\section{The MAIN RESUlts}

In the following, $\mathcal{X}$ will denote a subset of $L^{1}$ that contains the space of simple random variables $\mathcal{L}$ and $\rho: \mathcal{X} \rightarrow[-\infty, \infty]$. We say that $\rho$ is dilatation monotone whenever $\rho(\mathbb{E}[X \mid \pi]) \leq$ $\rho(X)$ for any $X \in \mathcal{X}$ and $\pi \in \Pi$ and that $\rho$ is law invariant if for any $X, Y \in \mathcal{X}$ such that $X, Y$ have the same distribution under $\mathbb{P}, \rho(X)=\rho(Y)$. $\rho$ is convex (resp., quasiconvex) whenever $\rho(\lambda X+(1-\lambda) Y) \leq \lambda \rho(X)+(1-\lambda) \rho(Y)$ (resp., $\rho(\lambda X+(1-\lambda) Y) \leq \max \{\rho(X), \rho(Y)\})$ for any $X, Y \in \mathcal{X}$ and $\lambda \in(0,1)$ such that $\lambda X+(1-\lambda) Y \in \mathcal{X} . \quad \rho$ is cash-additive whenever $\rho(X+s)=\rho(X)-s$ for any $X \in \mathcal{X}$ and $s \in \mathbb{R}$ with $X+s \in \mathcal{X} . \rho$ is monotone whenever $\rho(X) \leq \rho(Y)$ for any $X, Y \in \mathcal{X}$ with $X \geq Y$. We say that $\rho$ has the Fatou property if for any sequence $\left(X_{n}\right)_{n \in \mathbb{N}}$ in $\mathcal{X}$ and $X \in \mathcal{X}$ we have that

$$
X_{n} \stackrel{a . s .}{\longrightarrow} X \text { and } \sup _{n \in \mathbb{N}}\left|X_{n}\right| \in \mathcal{I}(\mathcal{X}) \Longrightarrow \rho(X) \leq \liminf _{n} \rho\left(X_{n}\right) .
$$

Let $\tau$ be a topology on $\mathcal{X}$. We say that $\rho$ is $\tau$ lower semicontinuous, whenever the sublevel set $\{\rho \leq \lambda\}:=\{X \in \mathcal{X}: \rho(X) \leq \lambda\}$ is $\tau$ closed for each $\lambda \in \mathbb{R}$. We also recall that if $\tau$ is a metrizable topology, then $\rho$ is $\tau$ lower semicontinuous if and only if for any sequence $\left(X_{n}\right)_{n \in \mathbb{N}}$ in $\mathcal{X}$ and $X \in \mathcal{X}$ we have that $X_{n} \stackrel{\tau}{\rightarrow} X$ implies $\rho(X) \leq \liminf _{n} \rho\left(X_{n}\right)$ (see, e.g., ([1], Lemma 2.42)). 
Remark 1. We note here that in the standard framework where $\mathcal{X}$ is a rearrangement invariant space, or more generally, an ideal of $L^{1}$, the supremum taken in $(\star)$ is an element of $\mathcal{X}$ and thus the definition of the Fatou property we give here coincides with the one used in the literature (see e.g. [13] and the references therein).

Convergence of finite conditional expectations plays a key role in the analysis of dilatation monotone functionals. It is a well-known fact that for any $X \in L^{1}$, one can construct a sequence $\left(\pi_{n}\right)_{n \in \mathbb{N}} \subset \Pi$ such that $\mathbb{E}\left[X \mid \pi_{n}\right] \stackrel{\text { a.s. }}{\longrightarrow} X$ and $\sup _{n \in \mathbb{N}}\left|\mathbb{E}\left[X \mid \pi_{n}\right]\right| \in L^{1}$. The following result tells us that we can pick $\left(\pi_{n}\right)_{n \in \mathbb{N}}$ in such a way that $\sup _{n \in \mathbb{N}}\left|\mathbb{E}\left[X \mid \pi_{n}\right]\right| \in \mathcal{I}(\{X, \mathbb{1}\})$, which enables us to give a positive answer to Question 2.8.2 in [2].

Lemma 2. For any $X \in L^{1}$, there exists $\left(\pi_{n}\right)_{n \in \mathbb{N}} \subset \Pi$ and $k \in \mathbb{R}_{+}$such that

$$
\mathbb{E}\left[X \mid \pi_{n}\right] \stackrel{\text { a.s. }}{\longrightarrow} X \text { and }\left|\mathbb{E}\left[X \mid \pi_{n}\right]\right| \leq|X|+k, \quad \text { for all } n \in \mathbb{N}
$$

Proof. Let $X \in L^{1}$ and $k_{1} \in \mathbb{R}_{+}$be such that $\mathbb{P}\left(|X| \leq k_{1}\right)>\frac{1}{2}$. Pick any $0<\epsilon<\frac{1}{2}$. Since $(\Omega, \mathcal{F}, \mathbb{P})$ is nonatomic, we can find $A \in \mathcal{F}$ such that $A \subset\left\{|X| \leq k_{1}\right\}$ and $\mathbb{P}(A)=\epsilon$. In addition, since $X \in L^{1}$, we can find $k_{2} \in \mathbb{R}$ such that $k_{2}>k_{1}$ and $\mathbb{E}\left[|X| \mathbb{1}_{|X|>k_{2}}\right]<\epsilon$. Put $\Omega^{\prime}=\left\{|X| \leq k_{2}\right\} \backslash A$ and note that $\mathbb{P}\left(\Omega^{\prime}\right)=\mathbb{P}\left(|X| \leq k_{2}\right)-\mathbb{P}(A) \geq \mathbb{P}\left(|X| \leq k_{1}\right)-\mathbb{P}(A)>0$. For the localized probability space $\left(\Omega^{\prime}, \mathcal{F}_{\mid \Omega^{\prime}}, \mathbb{P}_{\mid \Omega^{\prime}}\right)$, where $\mathcal{F}_{\mid \Omega^{\prime}}=\left\{E \in \mathcal{F}: E \subset \Omega^{\prime}\right\}$ and $\mathbb{P}_{\mid \Omega^{\prime}}(E)=\frac{\mathbb{P}(E)}{\mathbb{P}\left(\Omega^{\prime}\right)}$, we have that $X \mathbb{1}_{\Omega^{\prime}} \in L^{\infty}\left(\Omega^{\prime}, \mathcal{F}_{\mid \Omega^{\prime}}, \mathbb{P}_{\mid \Omega^{\prime}}\right)$. Applying $\left([12]\right.$, Lemma 3.1) to $L^{\infty}\left(\Omega^{\prime}, \mathcal{F}_{\mid \Omega^{\prime}}, \mathbb{P}_{\mid \Omega^{\prime}}\right)$, we can find a measurable partition $\left\{B_{1}, B_{2}, \ldots, B_{n}\right\}$ of $\Omega^{\prime}$ such that $\mathbb{P}\left(B_{i}\right)>0$ for each $i$ and

$$
\left|\sum_{i=1}^{n} \frac{\mathbb{E}\left[X \mathbb{1}_{B_{i}}\right]}{\mathbb{P}\left(B_{i}\right)} \mathbb{1}_{B_{i}}-X \mathbb{1}_{\Omega^{\prime}}\right|<\epsilon \mathbb{1}_{\Omega^{\prime}}
$$

Note that $\mathbb{P}\left(\Omega \backslash \Omega^{\prime}\right) \geq \mathbb{P}(A)=\epsilon>0$. Thus $\pi=\left\{B_{1}, B_{2}, \ldots, B_{n}, \Omega \backslash \Omega^{\prime}\right\} \in \Pi$. Applying (2.1), we obtain

$$
\begin{aligned}
\left|\mathbb{E}[X \mid \pi] \mathbb{1}_{\Omega^{\prime}}\right| & \leq\left|\mathbb{E}[X \mid \pi] \mathbb{1}_{\Omega^{\prime}}-X \mathbb{1}_{\Omega^{\prime}}\right|+\left|X \mathbb{1}_{\Omega^{\prime}}\right|=\mid \sum_{i=1}^{n} \frac{\mathbb{E}\left[X \mathbb{1}_{B_{i}}\right] \mathbb{1}_{B_{i}}-X \mathbb{1}_{\Omega^{\prime}}|+| X \mathbb{1}_{\Omega^{\prime}} \mid}{\mathbb{P}\left(B_{i}\right)} \\
& <\epsilon \mathbb{1}_{\Omega^{\prime}}+|X|<\frac{1}{2} \mathbb{1}_{\Omega^{\prime}}+|X| .
\end{aligned}
$$

Now, we will find an upper bound for $\left|\mathbb{E}[X \mid \pi] \mathbb{1}_{\Omega \backslash \Omega^{\prime}}\right|$. Note that

$$
\mathbb{E}\left[|X| \mathbb{1}_{\Omega \backslash \Omega^{\prime}}\right]=\mathbb{E}\left[|X| \mathbb{1}_{A \cup\left\{|X|>k_{2}\right\}}\right]=\mathbb{E}\left[|X| \mathbb{1}_{A}\right]+\mathbb{E}\left[|X| \mathbb{1}_{\left\{|X|>k_{2}\right\}}\right]<\epsilon\left(k_{1}+1\right) .
$$

In view of $\mathbb{P}\left(\Omega \backslash \Omega^{\prime}\right) \geq \epsilon$, we get that

$$
\left|\mathbb{E}[X \mid \pi] \mathbb{1}_{\Omega \backslash \Omega^{\prime}}\right| \leq \frac{\mathbb{E}\left[|X| \mathbb{1}_{\Omega \backslash \Omega^{\prime}}\right]}{\mathbb{P}\left(\Omega \backslash \Omega^{\prime}\right)} \mathbb{1}_{\Omega \backslash \Omega^{\prime}}<\left(k_{1}+1\right) \mathbb{1}_{\Omega \backslash \Omega^{\prime}}
$$

Therefore, by invoking (2.2), it follows that

$$
|\mathbb{E}[X \mid \pi]| \leq\left|\mathbb{E}[X \mid \pi] \mathbb{1}_{\Omega^{\prime}}\right|+\left|\mathbb{E}[X \mid \pi] \mathbb{1}_{\Omega \backslash \Omega^{\prime}}\right| \leq|X|+k_{1}+1 .
$$

We next claim that

$$
\|\mathbb{E}[X \mid \pi]-X\|_{1}<\epsilon\left(3+2 k_{1}\right) .
$$


Indeed, by applying (2.1) and (2.3), we get the following

$$
\begin{aligned}
\|\mathbb{E}[X \mid \pi]-X\|_{1} & =\left\|(\mathbb{E}[X \mid \pi]-X) \mathbb{1}_{\Omega^{\prime}}\right\|_{1}+\left\|(\mathbb{E}[X \mid \pi]-X) \mathbb{1}_{\Omega \backslash \Omega^{\prime}}\right\|_{1} \\
& \leq \epsilon+\left\|\mathbb{E}[X \mid \pi] \mathbb{1}_{\Omega \backslash \Omega^{\prime}}\right\|_{1}+\left\|X \mathbb{1}_{\Omega \backslash \Omega^{\prime}}\right\|_{1}=\epsilon+\left\|\mathbb{E}\left[X \mathbb{1}_{\Omega \backslash \Omega^{\prime}} \mid \pi\right]\right\|_{1}+\left\|X \mathbb{1}_{\Omega \backslash \Omega^{\prime}}\right\|_{1} \\
& \leq \epsilon+2\left\|X \mathbb{1}_{\Omega \backslash \Omega^{\prime}}\right\|_{1}<\epsilon\left(3+2 k_{1}\right) .
\end{aligned}
$$

Now letting, e.g., $\varepsilon=\frac{1}{n}$ in (2.4) and (2.5), we obtain $\left(\pi_{n}\right)_{n \in \mathbb{N}} \subset \Pi$ such that

$$
\sup _{n \in \mathbb{N}} \mid \mathbb{E}\left[X\left|\pi_{n}\right| \leq|X|+\left(k_{1}+1\right) \mathbb{1} \quad \text { and } \quad \mathbb{E}\left[X \mid \pi_{n}\right] \stackrel{\|\cdot\|_{1}}{\longrightarrow} X .\right.
$$

By passing to a subsequence, we may assume that $\mathbb{E}\left[X \mid \pi_{n}\right] \stackrel{a . s}{\longrightarrow} X$.

In general, the Fatou property is a weaker property than relative $\|\cdot\|_{1}$ lower semicontinuity. In particular, for $\mathcal{X}=L^{p}, 1 \leq p<+\infty$, the Fatou property is equivalent to $\|\cdot\|_{p}$ lower semicontinuity (see e.g. [14]). In the following proposition, we show that if $\rho$ is dilatation monotone, then the Fatou property is, in fact, equivalent to lower semicontinuity with respect to the relative $\sigma\left(L^{1}, \mathcal{L}\right)$ topology on $\mathcal{X}$.

Proposition 3. Let $\rho: \mathcal{X} \rightarrow[-\infty, \infty]$ be dilatation monotone. Then the following are equivalent

(i) $\rho$ has the Fatou property.

(ii) $\rho$ is lower semicontinuous with respect to the relative $\sigma\left(L^{1}, \mathcal{L}\right)$ topology on $\mathcal{X}$.

(iii) $\rho$ is lower semicontinuous with respect to the relative $\|\cdot\|_{1}$ topology on $\mathcal{X}$.

If any of the above holds, then for any $X \in \mathcal{X}$,

$$
\rho(X)=\sup _{\pi \in \Pi} \rho(\mathbb{E}[X \mid \pi])
$$

and for any $\left(\pi_{n}\right)_{n \in \mathbb{N}} \subset \Pi$,

$$
\mathbb{E}\left[X \mid \pi_{n}\right] \stackrel{\sigma\left(L^{1}, \mathcal{L}\right)}{\longrightarrow} X \Longrightarrow \rho(X)=\lim _{n} \rho\left(\mathbb{E}\left[X \mid \pi_{n}\right]\right)
$$

Proof. $(i) \Rightarrow($ ii $)$. Let $\{\rho \leq \lambda\}$ be a sublevel set and $\left(X_{\alpha}\right) \subset\{\rho \leq \lambda\}$ be any net such that $X_{\alpha} \stackrel{\sigma\left(L^{1}, \mathcal{L}\right)}{\longrightarrow} X \in \mathcal{X}$. By Lemma 2, we can find $k \in \mathbb{R}_{+}$and $\left(\pi_{m}\right)_{m \in \mathbb{N}} \subset \Pi$ such that $\mathbb{E}\left[X \mid \pi_{m}\right] \stackrel{\text { a.s. }}{\longrightarrow}$ $X$ and $\sup _{m \in \mathbb{N}}\left|\mathbb{E}\left[X \mid \pi_{m}\right]\right| \leq|X|+k$. By $(\star)$, we have that

$$
\rho(X) \leq \liminf _{m} \rho\left(\mathbb{E}\left[X \mid \pi_{m}\right]\right) .
$$

Note that for any $\pi=\left\{A_{1}, \ldots, A_{k}\right\} \in \Pi, \mathbb{E}\left[X_{\alpha} \mid \pi\right] \stackrel{\|\cdot\|_{1}}{\longrightarrow} \mathbb{E}[X \mid \pi]$. Indeed, we have that

$$
\begin{aligned}
\left\|\mathbb{E}\left[X_{\alpha} \mid \pi\right]-\mathbb{E}[X \mid \pi]\right\|_{1} & =\left\|\mathbb{E}\left[X-X_{\alpha} \mid \pi\right]\right\|_{1}=\left\|\sum_{i=1}^{k} \frac{\mathbb{E}\left[X_{\alpha} \mathbb{1}_{A_{i}}-X \mathbb{1}_{A_{i}}\right]}{\mathbb{P}\left(A_{i}\right)} \mathbb{1}_{A_{i}}\right\|_{1} \\
& \leq \mathbb{E}\left[\sum_{i=1}^{k}\left|\frac{\mathbb{E}\left[X_{\alpha} \mathbb{1}_{A_{i}}-X \mathbb{1}_{A_{i}}\right]}{\mathbb{P}\left(A_{i}\right)}\right| \mathbb{1}_{A_{i}}\right]=\sum_{i=1}^{k}\left|\mathbb{E}\left[X_{a} \mathbb{1}_{A_{i}}-X \mathbb{1}_{A_{i}}\right]\right| \rightarrow 0 .
\end{aligned}
$$

Thus, we can choose $\left(\alpha_{n}\right)_{n \in \mathbb{N}}$ such that $\left\|\mathbb{E}\left[X_{\alpha_{n}} \mid \pi\right]-\mathbb{E}[X \mid \pi]\right\|_{1} \leq \frac{1}{2^{n}}$ for all $n$. Then $Y:=$ $\sum_{k=1}^{\infty}\left|\mathbb{E}\left[X_{\alpha_{k}}-X \mid \pi\right]\right| \in L^{1}$. Note that for any $n \in \mathbb{N}, \sum_{k=1}^{n}\left|\mathbb{E}\left[X_{\alpha_{k}}-X \mid \pi\right]\right| \in \mathcal{R}_{\pi}$, the space of 
random variables measurable with respect to $\sigma(\pi)$. Since $\mathcal{R}_{\pi}$ is a finite dimensional subspace of $L^{1}$, it follows that $\mathcal{R}_{\pi}$ is a closed subspace of $L^{1}$ and thus $Y \in \mathcal{R}_{\pi}$. Moreover,

$$
\begin{aligned}
& \left|\mathbb{E}\left[X_{\alpha_{n}} \mid \pi\right]-\mathbb{E}[X \mid \pi]\right| \leq \sum_{k=n}^{\infty}\left|\mathbb{E}\left[X_{\alpha_{k}} \mid \pi\right]-\mathbb{E}[X \mid \pi]\right| \stackrel{\text { a.s. }}{\longrightarrow} 0, \\
& \sup _{n \in \mathbb{N}}\left\{\left|\mathbb{E}\left[X_{\alpha_{n}} \mid \pi\right]\right|\right\} \leq Y+|\mathbb{E}[X \mid \pi]| \in \mathcal{L} \subset \mathcal{X} \subset \mathcal{I}(\mathcal{X}) .
\end{aligned}
$$

Therefore, by applying again $(\star)$ to $\pi_{m}$, we get that, for any $m \in \mathbb{N}$,

$$
\rho\left(\mathbb{E}\left[X \mid \pi_{m}\right]\right) \leq \liminf _{n} \rho\left(\mathbb{E}\left[X_{\alpha_{n}} \mid \pi_{m}\right]\right) .
$$

Applying dilatation monotonicity of $\rho$ to the right hand side, we have that, for any $m \in \mathbb{N}$,

$$
\rho\left(\mathbb{E}\left[X \mid \pi_{m}\right]\right) \leq \liminf _{n} \rho\left(X_{\alpha_{n}}\right) .
$$

Thus, by (2.8), we get that $\rho(X) \leq \liminf _{n} \rho\left(X_{\alpha_{n}}\right) \leq \lambda$ and thus $X \in\{\rho \leq \lambda\}$.

$($ ii $) \Rightarrow($ iii $)$. This is immediate since the $\sigma\left(L^{1}, \mathcal{L}\right)$ topology is weaker than the $\|\cdot\|_{1}$ topology.

$($ iii $) \Rightarrow(i)$. Let $\left(X_{n}\right)_{n \in \mathbb{N}} \subset \mathcal{X}$ and $X \in \mathcal{X}$ be such that $X_{n} \stackrel{\text { a.s. }}{\longrightarrow} X$ and $\sup _{n \in \mathbb{N}}\left|X_{n}\right| \in \mathcal{I}(\mathcal{X})$.

By the Dominated Convergence Theorem, we have that $X_{n} \stackrel{\|\cdot\|_{1}}{\longrightarrow} X$ and (iii) yields that $\rho(X) \leq$ $\liminf _{n} \rho\left(X_{n}\right)$.

To verify (2.7), let $\mathbb{E}\left[X \mid \pi_{n}\right] \stackrel{\sigma\left(L^{1}, \mathcal{L}\right)}{\longrightarrow} X$ and note that by (ii) and the dilatation monotonicity of $\rho$, we have that $\rho(X) \leq \liminf _{n} \rho\left(\mathbb{E}\left[X \mid \pi_{n}\right]\right) \leq \limsup _{n} \rho\left(\mathbb{E}\left[X \mid \pi_{n}\right]\right) \leq \rho(X)$. In particular, it follows that $\rho(X)=\lim _{n} \rho\left(\mathbb{E}\left[X \mid \pi_{n}\right]\right)$. (2.6) follows by the dilatation monotonicity of $\rho$, (i) and Lemma 2 ,

Now, we turn to the extension problem. We are motivated by (2.6) to define the following functional associated with $\rho$.

$$
\bar{\rho}(X)=\sup _{\pi \in \Pi} \rho(\mathbb{E}[X \mid \pi]), \quad X \in L^{1} .
$$

Theorem 4. Let $\rho: \mathcal{X} \rightarrow(-\infty, \infty]$ be a dilatation monotone functional with the Fatou property. Then $\bar{\rho}: L^{1} \rightarrow(-\infty, \infty]$ is the unique extension of $\rho$ to $L^{1}$ that is dilatation monotone and $\sigma\left(L^{1}, \mathcal{L}\right)$ lower semicontinuous. Moreover, $\bar{\rho}$ is law-invariant and preserves monotonicity, (quasi)convexity, and cash-additivity of $\rho$.

Proof. In view of (2.6) , it is immediate to see that $\bar{\rho}$ agrees with $\rho$ on $\mathcal{X}$. Thus for any $X \in L^{1}$ and $\pi \in \Pi$, since $\mathbb{E}[X \mid \pi] \in \mathcal{L} \subset \mathcal{X}$, we get that

$$
\bar{\rho}(\mathbb{E}[X \mid \pi])=\rho(\mathbb{E}[X \mid \pi]) \leq \bar{\rho}(X) .
$$

This establishes dilatation monotonicity of $\bar{\rho}$ on $L^{1}$. Since $\rho>-\infty$ on $\mathcal{X}$, it also follows that $\bar{\rho}>-\infty$ on $L^{1}$. Next, we show that $\bar{\rho}$ is $\|\cdot\|_{1}$ lower semicontinuous, and thus by Proposition 3 , we get that $\bar{\rho}$ is $\sigma\left(L^{1}, \mathcal{L}\right)$ lower semicontinuous. Indeed, let $X_{n} \stackrel{\|\cdot\|_{1}}{\longrightarrow} X$ and fix some $\pi \in \Pi$. Then $\mathbb{E}\left[X_{n} \mid \pi\right] \stackrel{\|\cdot\|_{1}}{\longrightarrow} \mathbb{E}[X \mid \pi]$. Therefore, by the Fatou property of $\rho$ and the definition of $\bar{\rho}$,

$$
\rho(\mathbb{E}[X \mid \pi]) \leq \liminf _{n} \rho\left(\mathbb{E}\left[X_{n} \mid \pi\right]\right) \leq \liminf _{n} \bar{\rho}\left(X_{n}\right) .
$$

Taking supremum over $\pi$, we get that $\bar{\rho}(X) \leq \liminf _{n} \bar{\rho}\left(X_{n}\right)$ and thus $\bar{\rho}$ is $\|\cdot\|_{1}$ lower semicontinuous. The uniqueness of $\bar{\rho}$ is immediate by (2.6). 
The law-invariance of $\bar{\rho}$ follows by ([17], Theorem 18(ii)). Assume that $\rho$ is quasiconvex. Pick any $X_{1}, X_{2} \in L^{1}$ and $\lambda \in(0,1)$. Then

$$
\begin{gathered}
\bar{\rho}\left(\lambda X_{1}+(1-\lambda) X_{2}\right)=\sup _{\pi \in \Pi} \rho\left(\mathbb{E}\left[\lambda X_{1}+(1-\lambda) X_{2} \mid \pi\right]\right)=\sup _{\pi \in \Pi} \rho\left(\lambda \mathbb{E}\left[X_{1} \mid \pi\right]+(1-\lambda) \mathbb{E}\left[X_{2} \mid \pi\right]\right) \\
\leq \sup _{\pi \in \Pi} \max \left\{\rho\left(\mathbb{E}\left[X_{1} \mid \pi\right]\right), \rho\left(\mathbb{E}\left[X_{2} \mid \pi\right)\right\}\right) \leq \max \left\{\bar{\rho}\left(X_{1}\right), \bar{\rho}\left(X_{2}\right)\right\} .
\end{gathered}
$$

Hence, $\bar{\rho}$ is quasiconvex as well. In a similar manner, one shows that $\bar{\rho}$ preserves convexity, monotonicity and cash-additivity of $\rho$.

To present our applications to the study of quasiconvex law-invariant functionals, we need to apply the following result from [2]. In particular, the proof of ([2], Proposition 2.2) yields the following construction, which we isolate here.

Lemma 5. ([2]) For any $X \in L^{1}$ and $\pi \in \Pi$, there exists $k \in \mathbb{R}_{+}$and $X_{n, j}, j=1, \ldots, N_{n}, n \in \mathbb{N}$ such that $X_{n, j} \mathbb{1}_{\{|X| \leq n\}}$ has the same distribution as $X \mathbb{1}_{\{|X| \leq n\}}, X_{n, j} \mathbb{1}_{\{|X|>n\}}=X_{\{|X|>n\}}$ for each $j, n$ and

$$
\frac{1}{N_{n}} \sum_{j=1}^{N_{n}} X_{n, j} \stackrel{\text { a.s. }}{\longrightarrow} \mathbb{E}[X \mid \pi] \text { and }\left|\frac{1}{N_{n}} \sum_{j=1}^{N_{n}} X_{n, j}\right| \leq|X|+|\mathbb{E}[X \mid \pi]|+k, \quad \text { for all } n \in \mathbb{N} .
$$

Corollary 6. Let $\mathcal{X}$ be a convex subset of $L^{1}$ with the following properties

(i) $\mathcal{X}+\mathcal{X} \subset \mathcal{X}$

(ii) $L^{\infty} \subset \mathcal{X}$ and $X \mathbb{1}_{A} \in \mathcal{X}$ for each $X \in \mathcal{X}$ and $A \in \mathcal{F}$.

Then for any quasiconvex law-invariant $\rho: \mathcal{X} \rightarrow(-\infty, \infty]$ with the Fatou property, we have that $\bar{\rho}: L^{1} \rightarrow(-\infty, \infty]$, defined in (2.9), agrees with $\rho$ on $\mathcal{X}$, is quasiconvex, law-invariant, dilatation monotone and $\sigma\left(L^{1}, \mathcal{L}\right)$ lower semicontinuous. Moreover, $\bar{\rho}$ preserves monotonicity, convexity, and cash-additivity of $\rho$.

Proof. We will verify that $\rho$ is dilatation monotone, then the result follows by Theorem 4 . Let $X \in \mathcal{X}$ and $\pi \in \Pi$. Pick $X_{n, j}, j=1, \ldots, N_{n}, n \in \mathbb{N}$ as in Lemma 5. Then $X_{n, j} \mathbb{1}_{\{|X| \leq n\}} \in L^{\infty}$, and thus $X_{n, j}=X_{n, j} \mathbb{1}_{\{|X| \leq n\}}+X_{n, j} \mathbb{1}_{\{|X|>n\}}=X_{n, j} \mathbb{1}_{\{|X| \leq n\}}+X \mathbb{1}_{\{|X|>n\}} \in \mathcal{X}$. We also have that $X_{n, j}$ has the same distribution as $X$, therefore $\rho(X)=\rho\left(X_{n, j}\right)$ for each $j, n$ and by the quasi-convexity of $\rho$ we get that $\rho\left(\frac{1}{N_{n}} \sum_{j=1}^{N_{n}} X_{n, j}\right) \leq \rho(X)$. Finally by $(\star)$, it follows that $\rho(\mathbb{E}[X \mid \pi]) \leq \liminf _{n} \rho\left(\frac{1}{N_{n}} \sum_{j=1}^{N_{n}} X_{n, j}\right) \leq \rho(X)$.

Theorem 4 and Corollary 6 improve and extend some recent results in this topic (see e.g. ([20], Theorem 3.1) and ([17], Theorem 18, Proposition 20)) to domains $\mathcal{X}$ that are not necessarily linear nor rearrangement invariant. This general framework besides being of mathematical interest is also motivated by the Musielak-Orlicz spaces which are relevant to utility theory (see e.g. [1]).

In the following, we will derive a dual representation for convex $\rho$ in terms of the following conjugate function.

$$
\rho^{\#}(Y)=\sup _{X \in \mathcal{L}}\{\mathbb{E}[X Y]-\rho(X)\}, \quad Y \in L^{1} .
$$

We say that a subset $\mathcal{C}$ of $L^{1}$ is a dilatation monotone set if it is non-empty and $\mathbb{E}[X \mid \pi] \in \mathcal{C}$ for all $X \in \mathcal{C}$ and $\pi \in \Pi$. 
Lemma 7. Let $\rho: \mathcal{X} \rightarrow[-\infty, \infty]$ be dilatation monotone.

(i) $\rho^{\#}$ is dilatation monotone and $\|\cdot\|_{1}$ lower semicontinuous.

(ii) Let $\mathcal{C} \subset \mathcal{X}$ be a dilatation subset of $L^{1}$. If $\rho$ has the Fatou property, then for any $Y \in L^{1}$ such that $\mathbb{E}[|Y X|]<\infty$ for all $X \in \mathcal{C}$, we have that

$$
\sup _{X \in \mathcal{L} \cap \mathcal{C}}\{\mathbb{E}[X Y]-\rho(X)\}=\sup _{X \in \mathcal{C}}\{\mathbb{E}[X Y]-\rho(X)\} .
$$

Proof. (i) Fix any $Y \in L^{1}$ and $\pi \in \Pi$. We have that

$$
\begin{aligned}
\rho^{\#}(\mathbb{E}[Y \mid \pi]) & =\sup _{X \in \mathcal{L}}\{\mathbb{E}[X \mathbb{E}[Y \mid \pi]]-\rho(X)\}=\sup _{X \in \mathcal{L}}\{\mathbb{E}[Y \mathbb{E}[X \mid \pi]]-\rho(X)\} \\
& \leq \sup _{X \in \mathcal{L}}\{\mathbb{E}[Y \mathbb{E}[X \mid \pi]]-\rho(\mathbb{E}[X \mid \pi])\} \leq \sup _{Z \in \mathcal{L}}\{\mathbb{E}[Y Z]-\rho(Z)\}=\rho^{\#}(Y) .
\end{aligned}
$$

Thus $\rho^{\#}$ is dilatation monotone on $L^{1}$. Next, let $\left(Y_{n}\right)_{n \in \mathbb{N}} \subset L^{1}$ and $Y \in L^{1}$ such that $Y_{n} \stackrel{\|\cdot\|_{1}}{\longrightarrow} Y$. Then $\lim _{n}\left(\mathbb{E}\left[X Y_{n}\right]-\rho(X)\right)=\mathbb{E}[X Y]-\rho(X)$ for all $X \in \mathcal{L}$. Thus since $\rho^{\#}\left(Y_{n}\right) \geq \mathbb{E}\left[X Y_{n}\right]-\rho(X)$ for all $X \in \mathcal{L}$, it follows that $\liminf _{n} \rho^{\#}\left(Y_{n}\right) \geq \mathbb{E}[X Y]-\rho(X)$ for any $X \in \mathcal{L}$, implying that $\liminf _{n} \rho^{\#}\left(Y_{n}\right) \geq \rho^{\#}(Y)$. This proves that $\rho^{\#}$ is $\|\cdot\|_{1}$ lower semicontinuous.

(ii) It is clear that $c:=\sup _{X \in \mathcal{L} \cap \mathcal{C}}\{\mathbb{E}[X Y]-\rho(X)\} \leq \sup _{X \in \mathcal{C}}\{\mathbb{E}[X Y]-\rho(X)\}$. Let $Y \in L^{1}$ be such that $\mathbb{E}[|Y X|]<\infty$ for all $X \in \mathcal{C}$. Now pick any $X \in \mathcal{C}$. By Lemma 2, there exists a sequence $\left(\pi_{n}\right)_{n \in \mathbb{N}} \subset \Pi$ such that $\mathbb{E}\left[X \mid \pi_{n}\right] \stackrel{\text { a.s. }}{\longrightarrow} X$ and $X^{*}:=\sup _{n \in \mathbb{N}}\left\{\left|\mathbb{E}\left[X \mid \pi_{n}\right]\right|\right\} \in \mathcal{I}(\mathcal{C} \cup\{\mathbb{1}\})$, so that $\mathbb{E}\left[X^{*}|Y|\right]<\infty$. By the Dominated Convergence Theorem, $\mathbb{E}[Y X]=\lim _{n} \mathbb{E}\left[Y \mathbb{E}\left[X \mid \pi_{n}\right]\right]$. Thus since $c \geq \mathbb{E}\left[\mathbb{E}\left[X \mid \pi_{n}\right] Y\right]-\rho\left(\mathbb{E}\left[X \mid \pi_{n}\right]\right)$ for any $n \in \mathbb{N}$, by (2.7) we get that

$$
c \geq \lim _{n}\left(\mathbb{E}\left[\mathbb{E}\left[X \mid \pi_{n}\right] Y\right]-\rho\left(\mathbb{E}\left[X \mid \pi_{n}\right]\right)\right)=\mathbb{E}[X Y]-\rho(X) .
$$

Therefore, $c \geq \sup _{X \in \mathcal{C}}\{\mathbb{E}[X Y]-\rho(X)\}$, yielding the desired inequality.

Theorem 8. Let $\rho: \mathcal{X} \rightarrow(-\infty, \infty]$ be convex, dilatation monotone with the Fatou property, then $\bar{\rho}$ admits the following dual representation

$$
\bar{\rho}(X)=\sup _{Y \in \mathcal{L}}\left\{\mathbb{E}[X Y]-\rho^{\#}(Y)\right\}, \quad \text { for all } X \in L^{1} .
$$

If, moreover, $\rho$ is not identically equal to $\infty$, is cash-additive and monotone, then the dual representation can be simplified as follows

$$
\bar{\rho}(X)=\sup _{\frac{d \mathbb{Q}}{d \mathbb{P}} \in \mathcal{L}}\left\{\mathbb{E}_{\mathbb{Q}}[-X]-\rho^{\#}\left(-\frac{d \mathbb{Q}}{d \mathbb{P}}\right)\right\}, \quad \text { for all } X \in L^{1} .
$$

Proof. By Theorem 4, $\bar{\rho}: L^{1} \rightarrow(-\infty, \infty]$ is convex, dilatation monotone and $\sigma\left(L^{1}, \mathcal{L}\right)$ lower semicontinuous. Let $\bar{\rho}^{*}$ be the convex conjugate of $\bar{\rho}$, that is, $\bar{\rho}^{*}(Y)=\sup _{X \in L^{1}}\{\mathbb{E}[X Y]-\bar{\rho}(X)\}$, $Y \in \mathcal{L}$. By Lemma 7 (ii) applied with $\mathcal{C}=L^{1}$, it follows that

$$
\bar{\rho}^{*}(Y)=\sup _{X \in \mathcal{L}}\{\mathbb{E}[X Y]-\rho(X)\}=\rho^{\#}(Y),
$$

for all $Y \in \mathcal{L}$. By standard convex duality results (see, e.g., ([8], Proposition 4.1)) and (2.11), we get that

$$
\bar{\rho}(X)=\sup _{Y \in \mathcal{L}}\left\{\mathbb{E}[X Y]-\rho^{\#}(Y)\right\} .
$$

In the case where $\rho$ is not identically equal to $\infty$, is cash-additive and monotone it is standard to check that $\rho^{\#}(-Y)=\infty$ for all $Y \in \mathcal{L} \backslash \mathcal{D}$, where $\mathcal{D}=\left\{Y \in \mathcal{L}_{+}: \mathbb{E}[Y]=1\right\}$. Indeed, fix $X_{0} \in \mathcal{L}$ 
such that $\rho\left(X_{0}\right) \in \mathbb{R}$ and let $Y \in \mathcal{L}$ such that $\mathbb{E}[Y] \neq 1$, then we have that $\rho^{\#}(-Y) \geq \mathbb{E}\left[-Y\left(X_{0}+\right.\right.$ $m)]-\rho\left(X_{0}+m\right)=m(1-\mathbb{E}[Y])+\mathbb{E}\left[-Y X_{0}\right]-\rho\left(X_{0}\right)$ for all $m \in \mathbb{R}$, thus $\rho^{\#}(-Y)=\infty$. Suppose that $Y \geq 0$, then $\mathbb{E}\left[-Y \mathbb{1}_{\{Y<0\}}\right]>0$ and we have that $\rho^{\#}(-Y) \geq \mathbb{E}\left[-Y\left(X_{0}+m \mathbb{1}_{\{Y<0\}}\right)\right]-$ $\rho\left(X_{0}+m \mathbb{1}_{\{Y<0\}}\right) \geq m \mathbb{E}\left[-Y \mathbb{1}_{\{Y<0\}}\right]+\mathbb{E}\left[-Y X_{0}\right]-\rho\left(X_{0}\right)$ for all $m \in \mathbb{R}_{+}$, thus $\rho^{\#}(-Y)=\infty$. Therefore the representation (2.12) takes the form: $\bar{\rho}(X)=\sup _{Y \in \mathcal{L}}\left\{\mathbb{E}[-X Y]-\rho^{\#}(-Y)\right\}=$ $\sup _{Y \in \mathcal{D}}\left\{\mathbb{E}[-X Y]-\rho^{\#}(-Y)\right\}=\sup _{\frac{d \mathbb{Q}}{d \mathbb{P}} \in \mathcal{L}}\left\{\mathbb{E}_{\mathbb{Q}}[-X]-\bar{\rho}^{\#}\left(-\frac{d \mathbb{Q}}{d \mathbb{P}}\right)\right\}$.

We will now proceed to analyzing cash-additive hulls of dilatation monotone functionals. For $f: L^{1} \rightarrow(-\infty, \infty]$, recall that the cash-additive hull $\rho^{f}$ of $f$ introduced in [9] is defined as follows:

$$
\rho^{f}(X)=\inf _{s \in \mathbb{R}}\{f(X-s)-s\}, \quad X \in L^{1} .
$$

From the definition of $\rho^{f}$, it is clear that $\rho^{f}$ is cash-additive and is easy to see that $\rho^{f}$ preserves convexity, monotonicity and dilatation monotonicity of $f$. In the following result, we show that under a coercive condition, the infimum in (2.13) is attained, and moreover, $\rho^{f}$ preserves continuity properties of $f$.

Theorem 9. Let $f: L^{1} \rightarrow(-\infty, \infty]$ be dilatation monotone and $\|\cdot\|_{1}$ lower semicontinuous that satisfies the following coercive condition.

$$
\lim _{|s| \rightarrow \infty} f(s)+s=\infty
$$

Then $\rho^{f}: L^{1} \rightarrow(-\infty, \infty]$ is $\|\cdot\|_{1}$ lower semicontinuous and

$$
\rho^{f}(X)=\min _{s \in \mathbb{R}}\{f(X-s)-s\}, \quad \text { for all } X \in L^{1} .
$$

Proof. Pick any $X \in L^{1}$. If $\rho^{f}(X)=\infty$, then $f(X-s)-s=\infty$ for all $s \in \mathbb{R}$, and thus the infimum in (2.13) is attained at every $s \in \mathbb{R}$. Now assume that $\rho^{f}(X)<\infty$. Let $\left(s_{n}\right)_{n \in \mathbb{N}} \subset \mathbb{R}$ such that $f\left(X-s_{n}\right)-s_{n} \rightarrow \rho^{f}(X)$. Suppose that $\left(s_{n}\right)_{n \in \mathbb{N}}$ is unbounded. By passing to a subsequence, we may assume that $\left|s_{n}\right| \rightarrow \infty$. Since $f$ is dilatation monotone, we have

$$
f\left(X-s_{n}\right)-s_{n} \geq f\left(\mathbb{E}[X]-s_{n}\right)+\left(\mathbb{E}[X]-s_{n}\right)-\mathbb{E}[X],
$$

implying that

$$
\rho^{f}(X) \geq \underset{n}{\limsup }\left(f\left(\mathbb{E}[X]-s_{n}\right)+\left(\mathbb{E}[X]-s_{n}\right)\right)-\mathbb{E}[X] .
$$

Since $\left|\mathbb{E}[X]-s_{n}\right| \geq\left|s_{n}\right|-|\mathbb{E}[X]| \rightarrow \infty$, (2.14) implies that $\rho^{f}(X)=\infty$, which is a contradiction. Thus $\left(s_{n}\right)_{n \in \mathbb{N}}$ is bounded, and by passing to a subsequence, we may assume that $s_{n} \rightarrow s \in \mathbb{R}$. Therefore, $X-s_{n} \stackrel{\|\cdot\|_{1}}{\longrightarrow} X-s$. Since $f$ is $\|\cdot\|_{1}$ lower semicontinuous, we get that $f(X-s)-s \leq$ $\liminf _{n}\left(f\left(X-s_{n}\right)-s_{n}\right)=\rho^{f}(X)$. In particular, it follows that $\rho^{f}(X)=f(X-s)-s$, i.e., the infimum in (2.13) is attained, and consequently, $\rho^{f}(X) \neq-\infty$.

It remains to be shown that $\rho^{f}$ is $\|\cdot\|_{1}$ lower semicontinuous. Fix some $\lambda \in \mathbb{R}$ and let $\left(X_{n}\right)_{n \in \mathbb{N}} \subset L^{1}, X \in L^{1}$ be such that $X_{n} \stackrel{\|\cdot\|_{1}}{\longrightarrow} X$ and $\left(X_{n}\right)_{n \in \mathbb{N}} \subset\{\rho \leq \lambda\}$. Let $\left(s_{n}\right)_{n \in \mathbb{N}} \subset \mathbb{R}$ be such that $\rho^{f}\left(X_{n}\right)=f\left(X_{n}-s_{n}\right)-s_{n}$. Suppose that $\left(s_{n}\right)_{n \in \mathbb{N}}$ is unbounded. Then we can find a subsequence $\left(s_{n_{k}}\right)_{k \in \mathbb{N}}$ such that $\left|s_{n_{k}}\right| \rightarrow \infty$. Put $t_{k}=\mathbb{E}\left[X_{n_{k}}\right]-s_{n_{k}}$ and note that $\mathbb{E}\left[X_{n_{k}}\right] \rightarrow \mathbb{E}[X] \in \mathbb{R}$ and $\left|t_{k}\right| \geq\left|s_{n_{k}}\right|-\left|\mathbb{E}\left[X_{n_{k}}\right]\right| \rightarrow \infty$. Since $f$ is dilatation monotone, we have 
that $\rho^{f}\left(X_{n_{k}}\right)=f\left(X_{n_{k}}-s_{n_{k}}\right)-s_{n_{k}} \geq f\left(t_{k}\right)+t_{k}-\mathbb{E}\left[X_{n_{k}}\right]$. By (2.14), $f\left(t_{k}\right)+t_{k} \rightarrow \infty$, and thus $\lim _{k} \rho^{f}\left(X_{n_{k}}\right)=\infty$, which is a contradiction. Thus, $\left(s_{n}\right)_{n \in \mathbb{N}}$ is bounded, and we can extract a subsequence $\left(s_{n_{k}}\right)_{k \in \mathbb{N}}$ such that $s_{n_{k}} \rightarrow s \in \mathbb{R}$. Then $X_{n_{k}}-s_{n_{k}} \stackrel{\|\cdot\|_{1}}{\longrightarrow} X-s$. Since $f$ is $\|\cdot\|_{1}$ lower semicontinuous, we have that $f(X-s) \leq \liminf _{k} f\left(X_{n_{k}}-s_{n_{k}}\right)$ and that

$$
\begin{aligned}
\rho^{f}(X) & \leq f(X-s)-s \leq \liminf _{k} f\left(X_{n_{k}}-s_{n_{k}}\right)+\lim _{k}\left(-s_{n_{k}}\right) \\
& =\liminf _{k}\left(f\left(X_{n_{k}}-s_{n_{k}}\right)-s_{n_{k}}\right)=\liminf _{k} \rho^{f}\left(X_{n_{k}}\right) \leq \lambda .
\end{aligned}
$$

Therefore, $X \in\{\rho \leq \lambda\}$ and $\rho$ is $\|\cdot\|_{1}$ lower semicontinuous.

\section{TRANSFORMED NORM RISK MEASURES}

In this section, we will present our application to the study of transformed norm risk measures. We will follow the terminology and notations of [3, 4. We call a function $G:[0, \infty) \rightarrow[0, \infty]$ an Orlicz function if it left-continuous, convex, $\lim _{x \rightarrow 0^{+}} G(x)=G(0)=0$ and $\lim _{x \rightarrow \infty} G(x)=\infty$. Under these assumptions $G$ is increasing (i.e., $x \leq y \Longrightarrow G(x) \leq G(y)$ ). The convex conjugate $G^{*}$ of $G$ is again an Orlicz function. The Orlicz space corresponding to $G$ is given by $L^{G}=$ $\left\{X \in L^{1}: \mathbb{E}[G(c|X|)]<\infty\right.$ for some $\left.c>0\right\}$ and the Orlicz heart corresponding to $G$ is given by $M^{G}=\left\{X \in L^{G}: \mathbb{E}[G(c|X|)]<\infty\right.$ for any $\left.c>0\right\}$. The Luxemburg norm is given by the following formula

$$
\|X\|_{G}=\inf \left\{\lambda>0: \mathbb{E}\left[G\left(\frac{|X|}{\lambda}\right)\right] \leq 1\right\} .
$$

Recall that the Orlicz space has the equivalent description $L^{G}=\left\{X \in L^{1}:\|X\|_{G}<\infty\right\}$. Also, note that $G^{-1}(1):=\sup \{t>0: G(t) \leq 1\}=\frac{1}{\|\mathbb{1}\|_{G}}$. Finally, the Orlicz norm is given by the formula $\|X\|_{G}^{*}=\sup \left\{\mathbb{E}[X Y]:\|Y\|_{G} \leq 1\right\}$.

Let $F$ be a left-continuous, increasing, convex function from $[0, \infty)$ to $(-\infty, \infty]$, not identical equal to $\infty$, such that $\lim _{x \rightarrow \infty} F(x)=\infty, G$ a real-valued Orlicz function, and $H: \mathbb{R} \rightarrow[0, \infty)$ an increasing, convex function with $\lim _{x \rightarrow \infty} H(x)=\infty$. We denote with $F^{*}$ and $H^{*}$ the convex conjugate of $F$ and $H$ respectively. We will also make use of the following conditions introduced in [3, 4].

$$
\begin{array}{cc}
(F G H 1) & F\left(\frac{H(s)+\epsilon}{G^{-1}(1)}\right)<\infty \text { for some } s \in \mathbb{R} \text { and } \epsilon>0 . \\
(F G H 2) & \lim _{s \rightarrow \infty}\left(F(H(s))-G^{-1}(1) s\right)=\infty .
\end{array}
$$

The transformed norm risk measure $T$ is defined as follows

$$
T(X)=\inf _{s \in \mathbb{R}}\left\{F\left(\|H(s-X)\|_{G}\right)-s\right\} .
$$

The risk measure $T$ is well-defined on the following subset of $L^{1}$ :

$$
\mathcal{X}=\left\{X \in L^{1}: H(s-X) \in L^{G} \text { for all } s \in \mathbb{R}\right\} .
$$

It is easy to see that $\mathcal{X}$ is convex and $L^{\infty} \subset \mathcal{X}$. Moreover, $T: \mathcal{X} \rightarrow[-\infty, \infty]$ is monotone, convex and cash-additive. The following example illustrates that $\mathcal{X}$ is not a vector space in general. 
Example 10. For $H(x)=x^{+}, \mathcal{X}=L^{G}+L_{+}^{1}$. Indeed, let $X \in L^{G}, Y \in L_{+}^{1}$ and $s \in \mathbb{R}$. Then $(s-X)^{+} \in L^{G}$, and since $(s-X-Y)^{+} \leq(s-X)^{+},(s-X-Y)^{+} \in L^{G}$, proving that $L^{G}+L_{+}^{1} \subset \mathcal{X}$. Now let $X \in \mathcal{X}$. Then $(s-X)^{+} \in L^{G}$, and $X=s-(s-X)^{+}+(s-X)^{-} \in L^{G}+L_{+}^{1}$.

In [3, 4], an extensive analysis of the properties of $T$ has been carried out under the assumption that $T$ is restricted on $M^{\Phi}$, where $\Phi:=G \circ H_{0}$ and $H_{0}(x)=H(x)-H(0)$ for $x \geq 0$. We aim here to investigate the properties of $T$ without imposing any restrictions on the domain. To apply the results of the previous section, we will follow [3, 4] and view $T$ as a cash-additive hull $\rho^{f}$ of an appropriate functional $f$. We define $\bar{F}:[0, \infty] \rightarrow(-\infty, \infty]$ as follows:

$$
\bar{F}(x)= \begin{cases}F(x), & x \in[0, \infty) \\ \infty, & x=\infty\end{cases}
$$

and we put

$$
f(X)=\bar{F}\left(\|H(-X)\|_{G}\right), \quad X \in L^{1} .
$$

Lemma 11. Let $f$ as in (3.3). Then

(i) $f$ is convex, monotone, dilatation monotone, $\|\cdot\|_{1}$ lower semicontinuous on $L^{1}$.

(ii) Under (FGH2), we have that $\lim _{|s| \rightarrow \infty} f(s)+s=\infty$.

Proof. (i) Convexity and monotonicity of $f$ is clear. We assert that $f$ is dilatation monotone. Let $X \in L^{1}$ and $\pi \in \Pi$. If $H(-X) \notin L^{G}$, then $f(X)=\infty$ and $f(\mathbb{E}[X \mid \pi]) \leq f(X)$. Suppose that $H(-X) \in L^{G}$. Then by the conditional Jensen's inequality and ([7], Corollary 2.3.11),

$$
\begin{gathered}
0 \leq H(\mathbb{E}[-X \mid \pi]) \leq \mathbb{E}[H(-X) \mid \pi], \\
\|H(\mathbb{E}[-X \mid \pi])\|_{G} \leq\|\mathbb{E}[H(-X) \mid \pi]\|_{G} \leq\|H(-X)\|_{G} .
\end{gathered}
$$

Since $\bar{F}$ is increasing, we conclude that $f(\mathbb{E}[X \mid \pi]) \leq f(X)$ as desired.

Next, we show that $f$ is $\|\cdot\|_{1}$ lower semicontinuous. Let $\lambda \in \mathbb{R}$ and $\left(X_{n}\right)_{n \in \mathbb{N}} \subset\{f \leq \lambda\}$ be such that $X_{n} \stackrel{\|\cdot\|_{1}}{\longrightarrow} X \in L^{1}$. By passing to a subsequence, we may assume that $X_{n} \stackrel{\text { a.s. }}{\longrightarrow} X$ and $\sup _{n \in \mathbb{N}}\left\{\left|X_{n}\right|\right\} \in L^{1}$. Put $Y_{n}=\sup _{k \geq n} X_{k} \in L^{1}$ and note that $Y_{n} \downarrow X$. Since $H$ is continuous and increasing, $H\left(-Y_{n}\right) \uparrow H(-X)$. It follows that $\left\|H\left(-Y_{n}\right)\right\|_{G} \uparrow\|H(-X)\|_{G}$; indeed, if $\sup _{n \in \mathbb{N}}\left\{\left\|H\left(-Y_{n}\right)\right\|_{G}\right\}=\infty$, then the assertion is obvious, otherwise it follows from ([21], Theorem 131.6). Since $\bar{F}$ is left continuous, $\lim _{n} \bar{F}\left(\left\|H\left(-Y_{n}\right)\right\|_{G}\right)=\bar{F}\left(\|H(-X)\|_{G}\right)$. Now since $f\left(X_{n}\right) \geq f\left(Y_{n}\right)$ for each $n \in \mathbb{N}, f(X)=\lim _{n} f\left(Y_{n}\right) \leq \liminf _{n} f\left(X_{n}\right) \leq \lambda$, implying that $X \in\{\rho \leq \lambda\}$. This proves that $f$ is $\|\cdot\|_{1}$ lower semicontinuous.

(ii) We have that $f(s)+s=F\left(\|H(-s)\|_{G}\right)+s \geq F(0)+s$. Thus clearly, $\lim _{s \rightarrow \infty} f(s)+s=\infty$. We also have that $F\left(\|H(-s)\|_{G}\right)+s=F\left(\|\mathbb{1}\|_{G} H(-s)\right)+s=F\left(\frac{H(-s)}{G^{-1}(1)}\right)+s$, and consequently,

$$
\lim _{s \rightarrow-\infty} f(s)+s=\lim _{s \rightarrow \infty} F\left(\frac{H(s)}{G^{-1}(1)}\right)-s .
$$

Observe that for a convex function $V$ defined on $[0, \infty), \frac{V(s)-V(0)}{s}$ is increasing in $s$ and thus if $V(0) \in \mathbb{R}$ we get

$$
\lim _{s \rightarrow \infty} \frac{V(s)}{s}=\sup \left\{\frac{V(s)-V(0)}{s}: s \in[0, \infty)\right\}
$$


Applying this to $F$ and $H$ and using their monotonicity, we have

$$
\lim _{s \rightarrow \infty} \frac{F(s)}{s}=a, \lim _{s \rightarrow \infty} \frac{H(s)}{s}=b \text { for } a, b \in(0, \infty] .
$$

From this, it follows that for any $c \in(0, \infty)$,

$$
\lim _{s \rightarrow \infty} \frac{F\left(\frac{H(s)}{c}\right) c}{s}=\lim _{s \rightarrow \infty} \frac{F\left(\frac{H(s)}{c}\right)}{\frac{H(s)}{c}} \lim _{s \rightarrow \infty} \frac{\frac{H(s)}{c}}{\frac{s}{c}}=a b .
$$

In particular, $\lim _{s \rightarrow \infty} \frac{F(H(s))}{s}=a b$. We claim that $a b>G^{-1}(1)$. If $F$ takes $\infty$ value, then $a=\infty$ and thus $a b>G^{-1}(1)$. Suppose that $F$ is real-valued and $a b \leq G^{-1}(1)$. Then since $F \circ H$ is convex, we get that $\lim _{s \rightarrow \infty} \frac{F(H(s))}{s}=\sup \left\{\frac{F(H(s))-F(H(0))}{s}: s \geq 0\right\} \leq G^{-1}(1)$. Hence, $F(H(s))-G^{-1}(1) s \leq F(H(0))$ for each $s \geq 0$, which contradicts (FGH2). This proves the claim. Finally, we have that

$$
\begin{aligned}
\lim _{s \rightarrow \infty} F\left(\frac{H(s)}{G^{-1}(1)}\right)-s & =\lim _{s \rightarrow \infty} \frac{s}{G^{-1}(1)}\left(\frac{F\left(\frac{H(s)}{G^{-1}(1)}\right) G^{-1}(1)}{s}-G^{-1}(1)\right) \\
& =\lim _{s \rightarrow \infty} \frac{s}{G^{-1}(1)}\left(a b-G^{-1}(1)\right)=\infty .
\end{aligned}
$$

This completes the proof.

Let $\bar{T}$ be the extension of $T$ on $L^{1}$ given by formula (2.9). In the following results we show that $\bar{T}$ satisfies the axioms of convex risk measures and admits tractable representations.

Theorem 12. Suppose that (FGH2) holds. Then $\bar{T}$ is $\|\cdot\|_{1}$ lower semicontinuous, convex, monotone, cash-additive, dilatation monotone and is given by the following formula

$$
\bar{T}(X)=\inf _{s \in \mathbb{R}}\left\{\bar{F}\left(\|H(s-X)\|_{G}\right)-s\right\}, \quad X \in L^{1} .
$$

Proof. Let $f$ be as in (3.3). Note that the right hand side of (3.4) is $\rho^{f}$. It is clear that $\rho^{f}$ extends T. Also by Lemma 11 and Theorem 9, $\rho^{f}: L^{1} \rightarrow(-\infty, \infty]$ is dilatation monotone and $\|\cdot\|_{1}$ lower semicontinuous. Therefore by Proposition 3 and Theorem 4 , we have that $\bar{T}(X)=\rho^{f}(X)$ for all $X \in L^{1}$, also $\bar{T}$ preserves the convexity, monotonicity, and cash-additivity of $T$.

Corollary 13. Under the assumptions (FGH1) and (FGH2), we have that

$$
\bar{T}(X)=\sup _{\frac{d \mathbb{Q}}{d \mathbb{P}} \in \mathcal{L}}\left\{\mathbb{E}_{\mathbb{Q}}[-X]-T^{\#}\left(-\frac{d \mathbb{Q}}{d \mathbb{P}}\right)\right\}, \quad \text { for all } X \in L^{1},
$$

where

$$
T^{\#}\left(-\frac{d \mathbb{Q}}{d \mathbb{P}}\right)=\min _{\eta \in L_{+}^{G^{*}},\{\eta=0\} \subset\left\{\frac{d \mathbb{Q}}{d \mathbb{P}}=0\right\}}\left\{\mathbb{E}\left[\eta H^{*}\left(\frac{1}{\eta} \frac{d \mathbb{Q}}{d \mathbb{P}} \mathbb{1}_{\left\{\frac{d \mathbb{Q}}{d \mathbb{P}}>0\right\}}\right)\right]+F^{*}\left(\|\eta\|_{G}^{*}\right)\right\}
$$

Proof. First we note that (FGH1) ensures that $T$ is not identical equal to $\infty$ (see e.g. ([4], Lemma 5.2)). By Theorem 12 and Theorem 8 , we have that

$$
\bar{T}(X)=\sup _{\frac{d \mathbb{Q}}{d \mathbb{P}} \in \mathcal{L}}\left\{\mathbb{E}_{\mathbb{Q}}[-X]-T^{\#}\left(-\frac{d \mathbb{Q}}{d \mathbb{P}}\right)\right\}, \quad \text { for all } X \in L^{1},
$$

where $T^{\#}$ is given by the formula (2.10), that is $T^{\#}\left(-\frac{d \mathbb{Q}}{d \mathbb{P}}\right)=\sup _{X \in \mathcal{L}}\left\{\mathbb{E}_{\mathbb{Q}}[-X]-T(X)\right\}$. By equation (4.2) in [4] and (4], Theorem 5.1) we have that 


$$
\sup _{X \in M^{\Phi}}\left\{\mathbb{E}_{\mathbb{Q}}[-X]-T(X)\right\}=\min _{\eta \in L_{+}^{G^{*}},\{\eta=0\} \subset\left\{\frac{d \mathbb{Q}}{d \mathbb{P}}=0\right\}}\left\{\mathbb{E}\left[\eta H^{*}\left(\frac{1}{\eta} \frac{d \mathbb{Q}}{d \mathbb{P}} \mathbb{1}_{\left\{\frac{d \mathbb{Q}}{d \mathbb{P}}>0\right\}}\right)\right]+F^{*}\left(\|\eta\|_{G}^{*}\right)\right\},
$$

where $\Phi=G \circ H_{0}$ and $H_{0}(x)=H(x)-H(0)$, for $x \geq 0$. The result now follows by applying Lemma 7 (ii) with $\mathcal{C}=M^{\Phi}$.

Higher order dual risk measures. In this subsection, we will discuss the Kusuoka representations of higher order dual risk measures. We recall that the Average Value at Risk of $X \in L^{1}$ at level $\alpha \in(0,1]$ is given by the following formula

$$
\operatorname{AVaR}_{\alpha}(X)=\frac{1}{\alpha} \int_{0}^{\alpha} \operatorname{VaR}_{t}(X) \mathrm{d} t
$$

where $\operatorname{VaR}_{t}(X)=\inf \{m: \mathbb{P}(X+m<0) \leq t\}$. Also recall that $\mathrm{AVaR}_{\alpha}$ is cash-additive, dilatation monotone and $\|\cdot\|_{1}$ continuous.

In the following, fix some $p, c>1$ and put

$$
G(x)=x^{p}, H(x)=x^{+}, \quad F(x)=c x .
$$

Then the extended transformed norm risk measure is given by the following formula.

$$
\bar{T}_{c, p}(X)=\inf _{s \in \mathbb{R}}\left\{c\left\|(s-X)^{+}\right\|_{p}-s\right\}, \quad X \in L^{1},
$$

where $\|\cdot\|_{p}$ is the $L^{p}$-norm. $\bar{T}_{c, p}$ corresponds to the higher order dual risk measure studied in [6]. In this article, the authors derived a Kusuoka representation of $\bar{T}_{c, p}$ for $X \in L^{p}$. In the following corollary, we show that the representation holds for any $X \in L^{1}$.

Corollary 14. Let $\bar{T}_{c, p}$ be as in (3.6). Then $\bar{T}_{c, p}$ admits the following Kusuoka representation representation. For any $X \in L^{1}$,

$$
\bar{T}_{c, p}(X)=\sup _{\mu \in \mathcal{M}_{q}} \int_{0}^{1} \operatorname{AVaR}_{\alpha}(X) \mu(\mathrm{d} \alpha)
$$

where $q$ such that $\frac{1}{q}+\frac{1}{p}=1$ and $\mathcal{M}_{q}=\left\{\mu \in \mathcal{P}((0,1]): \int_{0}^{1}\left|\int_{\alpha}^{1} \frac{\mu(\mathrm{d} t)}{t}\right|^{q} \mathrm{~d} \alpha \leq c^{q}\right\}$.

Proof. It is straightforward to see that the condition (FGH2) is satisfied, thus by Theorem 12 we have that $\bar{T}_{c, p}$ is dilatation monotone and $\|\cdot\|_{1}$ lower semicontinuous. Let $X \in L^{1}$ and fix $\left(\pi_{n}\right)_{n \in \mathbb{N}} \subset \Pi$ such that $\mathbb{E}\left[X \mid \pi_{n}\right] \stackrel{\|\cdot\|_{1}}{\longrightarrow} X$. Then by (2.7), $\bar{T}_{c, p}(X)=\lim _{n} \bar{T}_{c, p}\left(\mathbb{E}\left[X \mid \pi_{n}\right]\right)$.

By the Kusuoka representation ([6], Theorem 1) and the dilatation monotonicity of $\mathrm{AVaR}_{\alpha}$, we get that

$$
\bar{T}_{c, p}\left(\mathbb{E}\left[X \mid \pi_{n}\right]\right)=\sup _{\mu \in \mathcal{M}_{q}} \int_{0}^{1} \operatorname{AVaR}_{\alpha}\left(\mathbb{E}\left[X \mid \pi_{n}\right]\right) \mu(\mathrm{d} \alpha) \leq \sup _{\mu \in \mathcal{M}_{q}} \int_{0}^{1} \operatorname{AVaR}_{\alpha}(X) \mu(\mathrm{d} \alpha) .
$$

Thus by letting $n \rightarrow \infty$, we get that

$$
\bar{T}_{c, p}(X) \leq \sup _{\mu \in \mathcal{M}_{q}} \int_{0}^{1} \operatorname{AVaR}_{\alpha}(X) \mu(\mathrm{d} \alpha)
$$

On the other hand, since $\operatorname{AVaR}_{\alpha}$ is $\|\cdot\|_{1}$ continuous, $\operatorname{AVaR}_{\alpha}(X)=\lim _{n} \operatorname{AVaR}_{\alpha}\left(\mathbb{E}\left[X \mid \pi_{n}\right]\right)$ for each $\alpha \in(0,1]$. Moreover, for each $n \in \mathbb{N}$, by the dilatation monotonicity of $\mathrm{AVaR}_{\alpha}$ applied to $\mathbb{E}\left[X \mid \pi_{n}\right]$

$$
\operatorname{AVaR}_{\alpha}\left(\mathbb{E}\left[X \mid \pi_{n}\right]\right) \geq \operatorname{AVaR}_{\alpha}\left(\mathbb{E}\left[\mathbb{E}\left[X \mid \pi_{n}\right]\right]\right)=\operatorname{AVaR}_{\alpha}(\mathbb{E}[X])=-\mathbb{E}[X]
$$


for each $\alpha \in(0,1]$, where the last equality is due to cash-additivity of $\mathrm{AVaR}_{\alpha}$. Now, fix some $\mu \in \mathcal{M}_{q}$. By Fatou's Lemma applied to the sequence $\left(\operatorname{AVaR} \bullet\left(\mathbb{E}\left[X \mid \pi_{n}\right]\right)\right)_{n}$,

$$
\int_{0}^{1} \operatorname{AVaR}_{\alpha}(X) \mu(\mathrm{d} \alpha) \leq \liminf _{n} \int_{0}^{1} \operatorname{AVaR}_{\alpha}\left(\mathbb{E}\left[X \mid \pi_{n}\right]\right) \mu(\mathrm{d} \alpha) .
$$

By using again the Kusuoka representation ([6], Theorem 1), we get that

$$
\begin{aligned}
\int_{0}^{1} \operatorname{AVaR}_{\alpha}(X) \mu(d \alpha) & \leq \liminf _{n} \sup _{\mu \in \mathcal{M}_{q}} \int_{0}^{1} \operatorname{AVaR}_{\alpha}\left(\mathbb{E}\left[X \mid \pi_{n}\right]\right) \mu(d \alpha)=\liminf _{n} \bar{T}_{c, p}\left(\mathbb{E}\left[X \mid \pi_{n}\right]\right) \\
& =\bar{T}_{c, p}(X) .
\end{aligned}
$$

Taking supremum over $\mathcal{M}_{q}$, we conclude that

$$
\sup _{\mu \in \mathcal{M}_{q}} \int_{0}^{1} \operatorname{AVaR}_{\alpha}(X) \mu(d \alpha) \leq \bar{T}_{c, p}(X) .
$$

This completes the proof.

Acknowledgements. The authors would like to thank Niushan Gao for valuable comments that improved the exposition of the manuscript.

\section{REFERENCES}

[1] C. D. Aliprantis and K. C. Border. Infinite Dimensional Analysis: A Hitchhiker's Guide, 3rd edn. Springer, 2006.

[2] S. Chen, N. Gao, and F. Xanthos. The strong Fatou property of risk measures. Dependence Modeling, 6(1):183-196, 2018.

[3] P. Cheridito and T. Li. Dual characterization of properties of risk measures on Orlicz hearts. Mathematics and Financial Economics, 2(1):29-55, 2008.

[4] P. Cheridito and T. Li. Risk measures on Orlicz hearts. Mathematical Finance, 19(2):189-214, 2009.

[5] A. S. Cherny and P. G. Grigoriev. Dilatation monotone risk measures are law invariant. Finance and Stochastics, 11(2):291-298, 2007.

[6] D. Dentcheva, S. Penev, and A. Ruszczyński. Kusuoka representation of higher order dual risk measures. Annals of Operations Research, 181(1):325-335, 2010.

[7] G. A. Edgar and L. Sucheston. Stopping Times and Directed Processes, volume 47. Cambridge University Press, 1992.

[8] I. Ekeland and R. Temam. Convex Analysis and Variational Problems, volume 28. SIAM, 1999.

[9] D. Filipović and M. Kupper. Monotone and cash-invariant convex functions and hulls. Insurance: Mathematics and Economics, 41(1):1-16, 2007.

[10] D. Filipović and G. Svindland. The canonical model space for law-invariant convex risk measures is $L^{1}$. Mathematical Finance, 22(3):585-589, 2012.

[11] M. Frittelli and M. Maggis. Conditional certainty equivalent. International Journal of Theoretical and Applied Finance, 14(1):41-59, 2011.

[12] N. Gao, D. Leung, C. Munari, and F. Xanthos. Fatou property, representations, and extensions of lawinvariant risk measures on general Orlicz spaces. Finance and Stochastics, 22(2):395-415, 2018.

[13] N. Gao and F. Xanthos. On the C-property and $w^{*}$-representations of risk measures. Mathematical Finance, 28(2):748-754, 2018.

[14] M. Kaina and L. Rüschendorf. On convex risk measures on $L_{p}$-spaces. Mathematical Methods of Operations Research, 69(3):475-495, 2009.

[15] P. Koch-Medina and C. Munari. Law-invariant risk measures: extension properties and qualitative robustness. Statistics \& Risk Modeling, 31(3-4):215-236, 2014.

[16] J. Leitner. Balayage monotonous risk measures. International Journal of Theoretical and Applied Finance, 7:887-900, 2004. 
[17] F.-B. Liebrich and G. Svindland. Efficient allocations under law-invariance: A unifying approach. Journal of Mathematical Economics, 84:28-45, 2019.

[18] T. Mao and R. Wang. Risk aversion in regulatory capital principles. SIAM Journal on Financial Mathematics, to appear.

[19] G. Svindland. Dilatation monotonicity and convex order. Mathematics and Financial Economics, 8(3):241$247,2014$.

[20] M. Tantrawan and D. H. Leung. On closedness of law-invariant convex sets in rearrangement invariant spaces. Archiv der Mathematik, 114:175-183, 2020.

[21] A. C. Zaanen. Riesz Spaces II, volume 30. Elsevier, 1983.

Department of Mathematics, Ryerson University, Toronto, Canada

E-mail address: srahnema@ryerson.ca

Department of Mathematics, Ryerson University, Toronto, Canada

E-mail address: foivos@ryerson.ca 